\title{
$\beta$ Adrenoceptor blockade and responses of serum lipids to a meal and to exercise
}

\author{
N R PEDEN, R J DOW， T E ISLES， B T MARTIN
}

\begin{abstract}
During a randomised placebo controlled trial of the effects of nadolol in hypertensive patients serum lipid profiles were obtained while the patients were fasting and during and after $a$ meal and an exercise test. Treatment with nadolol was associated with a significant reduction in high density lipoprotein cholesterol at all time points. Serum triglyceride concentrations during treatment with nadolol were higher in the fasting state, though not significantly so, increased further postprandially, and were significantly higher during and after exercise.

The changes in high density lipoprotein cholesterol and triglyceride concentrations during $\beta$ adrenoceptor blockade may be secondary to a reduction in lipoprotein lipase activity.
\end{abstract}

\section{Introduction}

$\beta$ Adrenoceptor antagonist drugs have been widely used in the long term management of patients with hypertension and angina and have more recently been licensed for use in secondary prevention after myocardial infarction. After a report that treatment with propranolol in hypertensive patients was associated with a significant increase in plasma triglyceride concentrations and a reduction in high density lipoprotein cholesterol concentration in the fasting state ${ }^{1}$ the question was raised whether these changes in lipoproteins might offset the advantage in coronary risk conferred by $\beta$ adrenoceptor blockade. ${ }^{2}$

The purpose of the present study was to examine the effects of non-selective $\beta$ adrenoceptor blockade on serum lipid concentrations in the fasted state, postprandially, and during exercise.

\section{Patients and methods}

Six non-obese, non-diabetic hypertensive patients (mean age 50 (range 40-68) who were not receiving any treatment gave informed consent to the study, which had the approval of the district ethical committee. A single blind randomised crossover design was used, each patient receiving nadolol $(40 \mathrm{mg}$ tablets) and a matching placebo for four weeks. For the first three days of each treatment phase half a tablet was taken daily; thereafter the patient took one tablet a day. After review at 14 days the dose was increased to two tablets a day. One patient felt very tired when the dose of nadolol was increased to $80 \mathrm{mg}$ a day and so took $40 \mathrm{mg}$ a day for the duration of active treatment.

At the end of each treatment phase patients attended the hospital, fasting, at 0800 . An intravenous cannula, which was kept flushed with saline, was inserted into a forearm vein. A blood sample was drawn at this time for measurement of the plasma nadolol concentra-

Departments of Pharmacology and Clinical Pharmacology and Biochemical Medicine, Ninewells Hospital and Medical School, Dundee

N R PEDEN, MA, MRCP, lecturer in clinical pharmacology

R J DOW, MB, MRCP, senior medical registrar

$T$ E ISLES, PHD, senior lecturer in biochemical medicine

B T MARTIN, PHD, senior biochemist

Correspondence to: Dr N R Peden, Falkirk and District Royal Infirmary, Falkirk FK1 5QE. tion, and the treatment tablets were then administered with a sip of water. After 30 minutes' rest the patients were given a standard breakfast consisting of fruit juice, boiled eggs, bread with butter and marmalade, and coffee with milk; this contained $64 \mathrm{~g}$ carbohydrate, $25 \mathrm{~g}$ fat, and $19 \mathrm{~g}$ protein, the total energy content being $2.35 \mathrm{MJ}$ $(562 \mathrm{kcal})$, of which $40 \%$ was fat. Thereafter patients remained recumbent for four hours. A graded exercise test was then performed on a bicycle ergometer, consisting of five minutes at $50 \%$ and five minutes at $75 \%$ of a previously determined maximal exercise capacity and thereafter continuing at maximal capacity until exhaustion. Blood samples were drawn before the meal and at $15,30,60,90,120,180$, and 240 minutes after the start of it, at the end of the exercise test, and after 10 minutes' rest.

Serum was separated and kept at $4^{\circ} \mathrm{C}$ for assay within 24 hours of concentrations of total cholesterol, ${ }^{3}$ high density lipoprotein cholesterol ${ }^{4}$ triglycerides, ${ }^{5}$ and glycerol ${ }^{6}$ by enzymatic methods modified for the Rotachem 2A centrifugal analyser (American Instrument Co). Low density lipoprotein cholesterol concentrations, in fasting specimens only, were calculated from the formula of Friedewald et al. ${ }^{\text {? }}$ Plasma nadolol concentrations were determined in samples kept frozen at $-20^{\circ} \mathrm{C}$ until they were assayed in a single batch. ${ }^{8}$
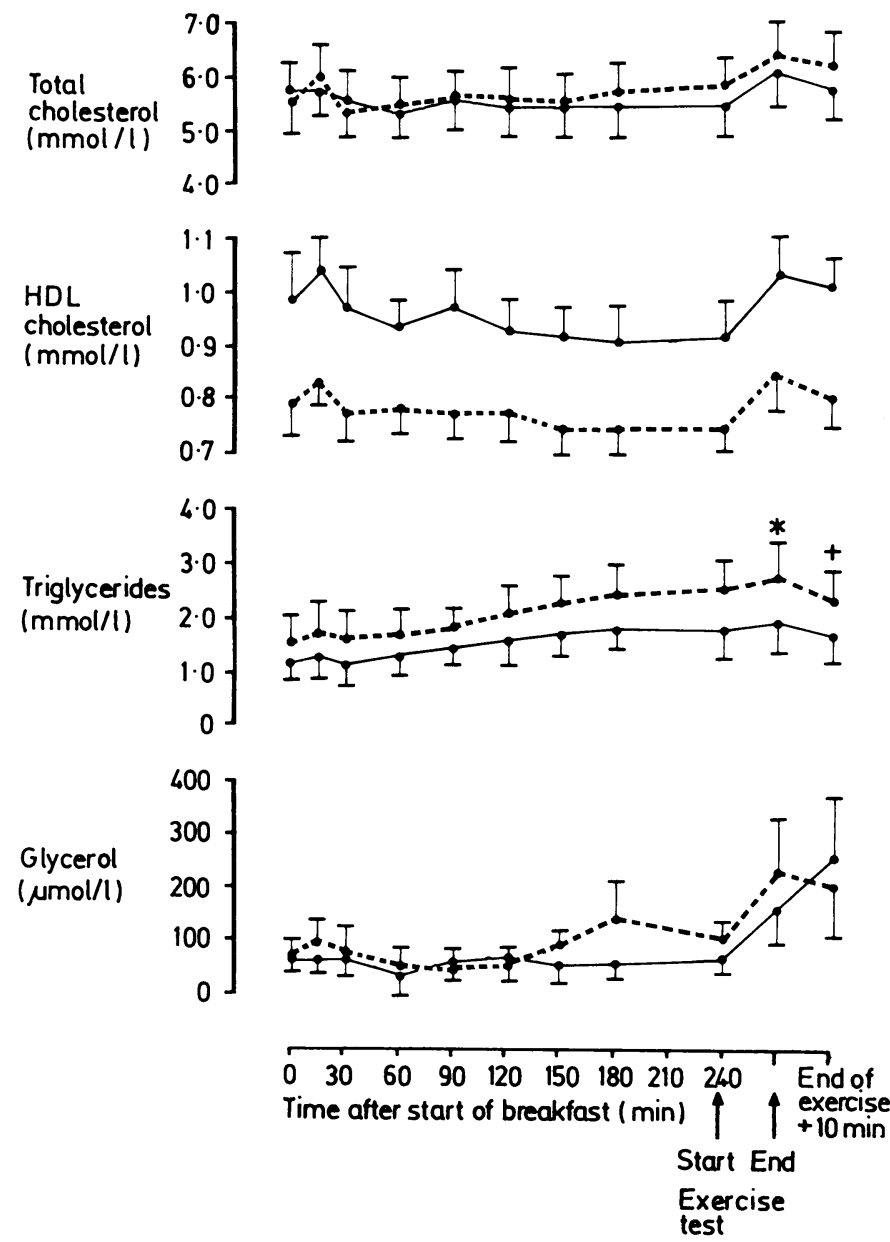

Serum total cholesterol, high density lipoprotein (HDL) cholesterol triglyceride, and glycerol concentrations during treatment with nadolol $(--\rightarrow)$ and placebo $(-)$.

Significance of difference between treatments: ${ }^{*} \mathrm{p}=0.042, \mathrm{p}=0.008$.

Conversion: SI to traditional units-Cholesterol: $1 \mathrm{mmol} / 1 \approx 38.7 \mathrm{mg} / 100$ $\mathrm{ml}$. Triglyceride: $1 \mathrm{mmol} / 1 \approx 88 \cdot 6 \mathrm{mg} / 100 \mathrm{ml}$. Glycerol: $1 \mu \mathrm{mol} / 1 \approx 9 \cdot 2 \mu \mathrm{g} /$ $100 \mathrm{ml}$. 
Statistical analysis was by analysis of variance for a two period crossover design. ${ }^{9}$ As the baseline concentration of high density lipoprotein cholesterol showed significant changes with treatment and this might have affected the responses at other time points, a further analysis of covariance was performed and a comparison made on adjusted means after correction for the baseline values. ${ }^{10}$ Program BMDP2V from the BMDP series statistical programs was used.11

\section{Results}

The figure shows the results. There were no significant differences in total cholesterol and glycerol concentrations between treatment with nadolol and with placebo at any time points. High density lipoprotein cholesterol concentrations, however, were significantly lower during treatment with nadolol at all time points (all $\mathrm{p}<0.03$ ). This was in part a reflection of a mean reduction of $20.4 \%$ in baseline concentrations, but after adjustment for this additional reductions in adjusted high density lipoprotein cholesterol concentrations were found 90 minutes after eating $(\mathrm{p}=0.007)$ and immediately after exercise $(p=0.029)$. Fasting concentrations of low density lipoprotein cholesterol were similar during treatment with placebo (mean (SEM) $4 \cdot 1$ $(0.5) \mathrm{mmol} / 1(159(19) \mathrm{mg} / 100 \mathrm{ml})$ ) and nadolol $(4.04(0.5) \mathrm{mmol} / \mathrm{l}$ (156 (19) $\mathrm{mg} / 100 \mathrm{ml})$ ).

Triglyceride concentrations were always higher during treatment with nadolol, but significance was not achieved in fasting specimens. The difference between treatment with placebo and nadolol became greater as the morning progressed and approached significance two and a half hours after the starting of the meal $(p=0.053)$. During exercise the difference became more pronounced, and triglyceride concentrations were significantly higher immediately after exercise $(p=0.042)$ and 10 minutes after exercise $(p=0.008)$ during treatment with nadolol compared with placebo.

The patient who received nadolol $40 \mathrm{mg}$ daily showed similar changes in high density lipoprotein cholesterol and triglyceride concentrations to the rest of the patients, who received $80 \mathrm{mg}$ daily.

Mean plasma nadolol concentrations before dosing were $51 \cdot 3$ (15.3) $\mu \mathrm{g} / \mathrm{l}$; peak concentrations were $151 \cdot 2(30 \cdot 2) \mu \mathrm{g} / \mathrm{l}$ and occurred 60-120 minutes after the start of breakfast.

\section{Discussion}

Catecholamine stimulation of hormone sensitive lipase and lipolysis in man appears to be mediated by $\beta_{1}$ and $\beta_{2}$ adrenoceptors. ${ }^{12}{ }^{13} \beta$ Adrenoceptor blockade might therefore be expected to inhibit lipolysis, reduce free fatty acid concentrations, and possibly increase serum triglyceride concentrations. It has, however, been suggested that the contribution of endogenous catecholamines to the maintenance of the resting rate of mobilisation of fat is slight. ${ }^{14}$ This, together with the uncontrolled nature of many studies and the differences in drugs used, dosages, and duration of treatment, may explain why data based on fasting blood specimens have given such conflicting results for several $\beta$ adrenoceptor blockers during long term treatment: a significant reduction ${ }^{15-17}$ or no change ${ }^{1819}$ in fasting free fatty acid concentrations and significant increases ${ }^{15172021}$ or no change ${ }^{181022-25}$ in fasting triglyceride concentrations have all been reported. In the present study, which was randomised and placebo controlled, we found a non-significant increase of $30 \%$ in fasting triglyceride concentrations during treatment with nadolol and no significant change in fasting glycerol concentrations.

In contrast to hormone sensitive lipase, lipoprotein lipase is an enzyme that is located on the endothelial surface of extrahepatic capillary beds; fasting triglyceride concentrations are inversely correlated with lipoprotein lipase activity, which itself correlates with the fractional removal rate of endogenous triglycerides. ${ }^{26}$ Treatment with propranolol may possibly be associated with a reduction in lipoprotein lipase activity, ${ }^{22}$ and this is also suggested by the finding that during long term treatment with a $\beta$ adrenoceptor blocker the rate of clearance of an exogenously administered triglyceride load (Intralipid) diminished. ${ }^{17}$ In a study of patients with hypertriglyceridaemia propranolol augmented the rise in triglyceride concentrations six hours postprandially. ${ }^{27}$ In the current study the difference in triglyceride concentrations between treatment with nadolol and with placebo increased postprandially, approaching significance. This may therefore represent the physiological counterpart of giving Intralipid with the $\beta$ adrenoceptor blocker inducing a reduction in clearance of an endogenous postprandial triglyceride load, perhaps due to a reduction in lipoprotein lipase activity.

During and shortly after recovery from strenuous exercise circulating catecholamine concentrations are high, ${ }^{28}$ and we found that triglyceride concentrations were significantly higher at the end of and after a strenuous exercise test during $\beta$ adrenoceptor blockade than during placebo treatment. Lipoprotein lipase activity increases during strenuous exercise, the increase correlating with urinary excretion of adrenaline. ${ }^{29}$ Inhibition of increases in lipoprotein lipase activity dependent on catecholamines during exercise may therefore explain the increase in triglyceride concentrations seen during treatment with nadolol.

Our findings in relation to changes in triglyceride concentrations postprandially and after exercise during $\beta$ adrenoceptor blockade suggest that failure to show changes in fasting triglyceride concentrations does not mean that such treatment may not have significant effects at other times of the day, depending on diet and activity. Increases in serum triglyceride concentrations provoked by long term $\beta$ adrenoceptor blockade might indeed be present for considerable periods of the day.

We also found that there was a significant reduction in high density lipoprotein cholesterol concentrations at all time points during treatment with nadolol. Reductions in these concentrations have previously been noted during treatment with propranolol, ${ }^{17}{ }^{12}$ oxprenolol, ${ }^{17}$ sotalol, ${ }^{16}$ atenolol, ${ }^{17}$ and metoprolol, ${ }^{17}$ though these have not been constant findings. ${ }^{1923-25}$ Treatment with the $\beta_{2}$ agonist terbutaline is associated with a rise in high density lipoprotein cholesterol concentrations. ${ }^{30}$ Cholesterol transfers to high density lipoprotein during catabolism of lipoprotein fractions rich in triglyceride, ${ }^{31}$ and high density lipoprotein cholesterol content correlates with the rate of catabolism of plasma triglycerides by lipoprotein lipase. ${ }^{32}$ Hence any factor reducing lipoprotein lipase activity and hence triglyceride catabolism might be expected to decrease high density lipoprotein cholesterol concentrations. An inverse relation between changes in concentrations of high density lipoprotein cholesterol and of very low density lipoprotein rich in triglycerides has previously been noted during long term $\beta$ adrenoceptor blockade ${ }^{172}$ and was confirmed indirectly in the present study.

It has recently been shown that high density lipoprotein cholesterol and its ratio to total cholesterol rises acutely within 10 minutes of the start of exercise with a bicycle ergometer and that this effect has disappeared by 15 minutes after exercise is stopped. ${ }^{33}$ Our results confirm this pattern of change during exercise and suggest that this rise is attenuated during $\beta$ adrenoceptor blockade. Triglyceride concentrations rose significantly after the start of the exercise test during treatment with nadolol, again suggesting that catabolism of triglycerides was reduced by the $\beta$ adrenoceptor blockade; this may explain the effect of nadolol on high density lipoprotein cholesterol concentrations during exercise.

In conclusion, we found significant reductions in serum high density lipoprotein cholesterol concentrations during treatment with nadolol, which were associated with increases in triglyceride concentrations. Increases in triglyceride concentrations were less pronounced in the fasting state than postprandially and during exercise. These changes may all be secondary to a reduction in lipoprotein lipase activity induced by $\beta$ adrenoceptor blockade. The pathological importance, if any, of a reduction in high density lipoprotein cholesterol concentrations in the absence of appreciable changes in low density lipoprotein cholesterol concentrations during long term $\beta$ adrenoceptor blockade remains unclear ${ }^{231}$ and will have to await further long term studies for clarification.

We thank Mr K F Yee for the statistical analysis; Mrs S Fitzpatrick 
for technical help; the department of medical illustration for the figure; Miss J Mitchell, dietetic department, for organising the standard meals; Mrs L Connolly for typing the manuscript; and Squibb (UK) for financial support.

\section{References}

' Leren P, Helgeland A, Holme I, Foss PO, Hjermann I, Lund-Larsen PG. Effect of propranolol and prazosin on blood lipids. The Oslo study. Lancet 1980;ii:

${ }^{2}$ Anonymous. Antihypertensive drugs, plasma lipids and coronary disease [Editorial ]. Lancet 1980;ii:19-20

of total serum cholesterol. Clin Chem $1974 ; 20: 470-5$.

- Berstein M, Scholnick HR, Morfin R. Rapid method for the isolation of lipo proteins from human serum by precipitation with polyamines. $\mathcal{J}$ Lipid Res 1970 $11: 583-95$.

${ }^{5}$ Bucolo G, David H. Quantitative determination of serum triglycerides by the use

of enzymes. Clin Chem 1973;19:476-82.
Wahlefeld AW. Triglycerides. Determination after enzymatic hydrolysis. In Bergmeyer HU, ed. Methods in enzymatic analyses. 2nd ed. London: Academic

' Friedewald WT, Levy RI, Fredrickson DS. Estimation of the concentration of lowdensity lipoprotein cholesterol in plasma without use of the preparative ultra-

Ivashnik E. Fluorometric determination of nadolol in human serum and urine. f Pharm Sci 1977;66:1168-72.

period cross-over clinical trial. Br $\mathcal{F}$ Clin Pharmaco

10 Cochran WG. Analysis of covariance: its nature and uses. Biometrics $1957 ; 13$ : 261-81.

Dixon WJ, Brown MB, eds. Biomedical computer programs $P$ series. London: University of California Press, 1979 .

2 Gibbons DO, Lant A, Ashford A, Collins RF, Pinder S. Comparative effects of acebutolol and practolol on the lipolytic response to isoprenaline. $\mathrm{Br} \mathcal{J}$ Clin Pharmacol 1976;3:177-84.

$13 \mathrm{Harms} \mathrm{HH}$, De Vente J, Zaagsma J. $\beta$-adrenoceptor blocking agents and lipolysis. Br $\mathcal{F}$ Clin Pharmacol 1982;13:181-6S.

14 Pinter EJ, Pattee CJ. Effect of $\beta$-adrenergic blockade on resting and stimulated

fat mobilisation $\mathrm{F}$ Clin Endocrinol Metab pay J, Simpson N, Metcalfe J, Page RL. Metabolic consequences of atenolol and
propranolol in treatment of essential hypertension. Br Med $\mathcal{f} 1979 ; \mathrm{i}: 77-80$.
16 Lehtonen A, Viikari J. Long-term effect of sotalol on plasma lipids. Clin Sci $1979 ; 57: 405-7 S$

17 Day JL, Metcalfe J, Simpson CN. Adrenergic mechanisms in control of plasma lipid concentrations. $\mathrm{Br}$ Med $\mathcal{f}$ 1982;284:1145-8.

${ }^{8}$ Nilsson A, Hanson BG, Hokfeldt B. Effect of metoprolol on blood glycerol, free fatty acids, triglycerides and glucose in relation to plasma catecholamines in hypertensive patients at rest and following submaximal work. Eur $\mathcal{f}$ Clin

Pharmacol 1978;13:5-8.
19irnbaum J, Di Bianco R, Becker KL, et al. Glucose and lipid metabolism during acebutolol and propranolol therapy of angina in non-diabetic patients. Clin

Pharmacol Ther 1983;33:294-300.
England JP, Hau AD, Shaw J. $\beta$-adrenoceptor blocking agents and lipid metabolism. Clin Sci Mol Med 1978;55:323-4S.

Waal-Manning HJ. Metabolic effects of $\beta$-adrenoceptor blockers. Drugs 1976; 11, suppl $1: 121-6$

administration 25:1071-5.

${ }^{23}$ Bauer JH, Brooks CS, Weinstein I, et al. Effects of diuretic and propranolol on plasma lipoprotein lipids. Clin Pharmacol Ther 1981;30:35-43.

${ }^{44}$ Kristensen BO. Effect of long-term treatment with beta blocking drugs on plasma

lipids and lipoproteins. $\mathrm{Br} M e d \mathcal{F} 1981 ; 283: 191-2$.
as Ballantyne P, Ballantyne $\mathrm{FC}, \mathrm{McMurdo} \mathrm{M}$. Effect of slow oxprenolol and a combination of slow oxprenolol and cyclopenthiazide on plasma lipoproteins. Atherosclerosis $1981 ; 39: 301-6$

${ }^{3}$ Huttunen JK, Ehnholm C, Kekki M, Nikkila EA. Post-heparin plasma lipoprotein lipase and hepatic lipase in normal subjects and in patients with hypertriglyceridaemia : correlations to sex, age and various parameters of triglyceride metabo-

27 Barboriak JJ, Friedberg HD. Propranolol and hypertryglyceridaemia. Atherosclerosis $1973 ; 17: 31-3$.

"8 Bloom SR, Johnston RH, Park DM, Rennie MJ, Sulaiman WR. Difference in the metabolic and hormonal response to exercise between racing cyclists and untrained individuals. $\mathcal{F}$ Physiol (Lond) 1976;258:1-18.

20 Lithell H, Cedermark M, Friberg J, Tesch P, Karlsson J. Increase of lipoprotein lipase activity in skeletal muscle during heavy exercise. Relation to epinephrine excretion. Metabolism 1981;30:1130-4.

${ }^{30}$ Hooper PL, Woo W, Visconti L, Pathak DR. Terbutaline raises high density lipoprotein cholesterol levels. $N$ Engl f Med $1981 ; 305: 1455-7$.

${ }^{31}$ Lewis B. The lipoproteins: predictors, protectors and pathogens. $\mathrm{Br} \mathrm{Med} \mathfrak{f}$ 1983;287:1161-4.

$32 \mathrm{Kekki} M$. Lipoprotein-lipase action determining high density lipoprotein cholesterol in adult normolipaemics. Atherosclerosis $1980 ; 37: 3-50$

3 Lennon DLF, Stratman FW, Shrago E, et al. Total cholesterol and HDL cholesmen and women.

(Accepted 11 April 1984)

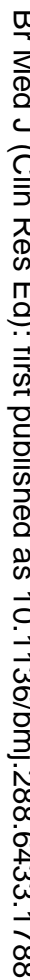

\title{
The dysplastic naevus syndrome and endocrine disease
}

\author{
S J ADAMS, MALCOLM H A RUSTIN, T W E ROBINSON, D D MUNRO
}

\begin{abstract}
Members of two different families were found to have the dysplastic naevus syndrome and coexistent endocrine abnormalities. The dysplastic naevus syndrome is probably inherited as an autosomal dominant trait and has been associated with other primary malignancies. This is the first time that it has been described in association with endocrine abnormalities.
\end{abstract}

\section{Introduction}

Dysplastic naevi are clinically and histologically distinct skin lesions that may be formal histogenetic precursors of cutaneous melanoma. First described by Cawley in 1952,1 they have subsequently been reported as the $\mathrm{BK}$ mole syndrome and the familial atypical mole malignant melanoma syndrome.

Department of Dermatology, University College Hospital, London WC1E 6AU

S J ADAMS, MB, MRCP, senior registrar

T W E ROBINSON, MA, FRCP, consultant dermatologist

Department of Dermatology, St Bartholomew's Hospital, London EC1

MALCOLM H A RUSTIN, BSC, MRCP, registrar

D D MUNRO, MD, FRCP, consultant dermatologist

Correspondence to: Dr S J Adams.
Characteristic features of the syndrome are a family history of malignant melanoma and multiple large melanocytic naevi with irregular outlines and variegated pigmentation. Histological examination shows a compound melanocytic naevus with atypical melanocytic hyperplasia, fibroplasia, new vessel formation, and a mixed lymphocytic and macrophage infiltrate in the dermis. Although the associated malignant melanoma is assumed to arise from the dysplastic naevi, MacKie observed melanoma arising in clinically normal skin and consequently suggested an alternative mechanism in the "activated and expanded melanocyte syndrome." 2

The dysplastic naevus syndrome is probably inherited as an autosomal dominant trait, and a link with other primary malignancies has been reported. ${ }^{3}$ An association with endocrine abnormalities has hitherto not been described, but we report here on two families with the dysplastic naevus syndrome and malignant melanoma who had coexistent multiple endocrine abnormalities.

\section{Case reports}

\section{FAMILY 1}

The eldest girl of four siblings was diagnosed as having multiple endocrine adenopathy with hyperparathyroidism and a pancreatic islet cell tumour. She died several years later from metastatic malignant melanoma after previous local excision of malignant melanoma. During a routine follow up of the family primary hyperparathyroidism was diagnosed in her twin sisters, one of whom had undergone 